\title{
Experience facilitates olfactory quality discrimination
}

\author{
MICHAEL D. RABIN \\ Yale University and John B. Pierce Foundation Laboratory, New Haven, Connecticut
}

\begin{abstract}
Perceptual learning in olfactory quality discrimination was investigated in two experiments. In Experiment 1, it was asked whether training participants to label target odorants would improve subsequent discrimination performance. Four groups participated. Prior to discrimination testing, one group was asked to provide a name for each of seven target odors and received training to ensure that reliable naming occurred (label training). A second group profiled the quality of the target odorants, using an odor-adjective attribute list (profile group). A third group was trained to label seven control odorants, and a fourth received no prior experience. Discrimination performance by each group ranked as follows: [label training on targets] $>$ [profile experience on targets] $>$ [label training on controls $=$ no prediscrimination experience]. Experiment 2 was designed to relate the odor knowledge a participant brought into the experiment to performance on a discrimination task wherein an odorant (the target) was paired either with itself or with a mixture consisting of target odorant plus a familiar or an unfamiliar contaminant (target transform). Six different targets were selected for each participant, to represent three familiar and three unfamiliar odors. Familiar targets and familiar contaminants facilitated discrimination. Taken together, these two experiments demonstrate that olfactory quality discrimination can be improved through training, or via experience naturally accumulated over time.
\end{abstract}

Discrimination can be measured in absolute or relative terms. Absolute discrimination typically refers to nonpaired comparisons of stimuli, as in identification or labeling. Identifying an odor emanating from a flask as "rose" is an example of absolute identification. Relative discrimination refers to an organism's ability to judge stimuli the same or different when perceiving them relative to one another. Judging the odor of a rose to be different from the odor of a violet is an example of relative discrimination. Presumably, this relative discrimination could be performed without knowledge of the odors' identities. Relative stimulus discriminability per se provides the theoretical limiter of absolute identification (Garner, 1962). Therefore, one would expect identification to provide only an indirect and, perhaps, conservative measure of sensory discrimination (Pollack, 1952).

The limits of odor-quality discrimination have not been studied directly with a relative discrimination procedure. Rather, odor-quality discrimination has been primarily investigated by means of odor-labeling techniques (Cain, 1979; Desor \& Beauchamp, 1974; Engen \& Pfaffman, 1960). Absolute identification experiments in olfaction have led to the conclusion that experience with the test

This research was supported by a grant from The Fragrance Research Fund and by National Institutes of Health Grant NS 21644 . This article is based on a doctoral dissertation presented to the Department of Psychology, Yale University. I thank William S. Cain for the nutelage and encouragement necessary for the completion of this work. I also thank Robert Crowder, Wendell Garner, William Kessen, and Lawrence Marks for their help and advice. Correspondence should be addressed to Michael D. Rabin, Research and Development, International Flavors and Fragrances, 1515 Highway 36, Union Beach, NJ 07735. stimuli can overcome encoding and retrieval difficulties that might limit estimates of channel capacity.

Perceptual learning "refers to an increase in the ability to extract information from the environment, as a result of experience and practice with stimulation coming from it" (Gibson, 1969, p. 3). If perceptual learning occurs in a discrimination experiment, the participants in the experiment should exhibit improved responses to differences between stimuli. Interestingly, perceptual learning has been demonstrated in various modalities for relative discrimination tasks (Gibson \& Walk, 1956; Pick, 1965; Robinson, 1955). Hence, even relative discrimination can be improved by experimental manipulation or training.

Laboratory experiments have sought to isolate the variables that contribute to improved discrimination. Label training has emerged as a functional method for improving certain types of discrimination. Gibson (1969) suggested that verbal labels focus attention on the discriminative features or invariant patterns already present in the stimulus. Gibson also pointed out that verbal labels might increase the efficiency of remembering, as might be required when stimuli are, often of necessity, presented successively. So, labels may draw attention to the definitive attributes of a stimulus, and may be necessary if the discrimination involves nonsimultaneous presentation of a standard and a comparison stimulus (see e.g., Murray \& Lee, 1977).

The following experiments were performed in order to examine perceptual learning in olfaction. The first experiment involved successive discrimination between odors of single chemical compounds. The second involved discrimination between simultaneous odor presentations- 
that is, the ability to discriminate between the components of an odor mixture.

\section{EXPERIMENT 1}

In Experiment 1, quality discrimination in a twointerval same-different task was investigated. It was asked whether label training or perhaps just experience with odors prior to discrimination testing can lead to increased ability to tell the odors apart.

\section{Method}

\section{Participants}

Fifty-six adults participated in this experiment. The participants were randomly divided into four groups that comprised 7 males and 7 females each. Most of the participants were students in Introductory Psychology, who received course credit for their time. A smaller number, who were paid for their time, were distributed equally among the four experimental groups.

\section{Stimuli}

Seven reagent-grade chemical odorants were matched for perceived intensity and chosen to represent low initial familiarity and relatively neutral pleasantness, based on preliminary experimentation with 10 other subjects. The odorants were matched to a moderate intensity level so that their intensity could not provide a cue for discrimination; the participants would thereby be forced to rely upon quality differences.

Human beings tend to base similarity judgments on the pleasantness of simple chemical stimuli (Schiffman, Robinson, \& Erickson, 1977). Therefore, it was desirable to select stimuli that were not distinctive in terms of pleasantness, so that their initial discriminability would not be facilitated by a large hedonic range. Familiarity was restricted to low average values, to allow learningshould it take place-within the appropriate experimental groups.

The following criteria were used to select the odorants for this experiment: (1) the odorants had to be of equal moderate intensity, (2) the odorants had to be near neutral in hedonic tone, and (3) the odorants had to be below average in familiarity. These criteria are not easy to meet, because of the correlation between familiarity and pleasantness (Engen \& Ross, 1973; Lawless \& Cain, 1975), but appropriate stimuli were found.

Mineral oil served as the diluent for all the odorants. The following odorants were selected: amyl butyrate (AB), $0.41 \%$ (v/v) (Monsanto); butyl alcohol (BA), 1.22\% (Mallinckrodt); cinnamyl n-butyrate (CB), 0.14\% (Pfaltz \& Bauer); octanal (OC), 0.02\% (Pfaltz \& Bauer); sec phenethyl alcohol (SP), $0.56 \%$ (Pfaltz \& Bauer); propyl butyrate, 0.14\% (Eastman); trans-2-decen-1-al (TD), $0.06 \%$ (Pfaltz \& Bauer).

\section{Stimulus Delivery}

A unique stimulus-delivery method was devised for these experiments. The odorants were injected into Interflo pellets (Chromex Corp., No. P-375), which are made of compressed filaments of polypropelyne. Interflo acts as an excellent adsorbent, capable of holding a relatively large amount of odorant and releasing it slowly over time. Each cylindrical pellet, which measured approximately $1 \mathrm{~cm}$ in length by $1 \mathrm{~cm}$ in diameter, could retain up to approximately $0.2 \mathrm{cc}$ of fluid. For both experiments, $0.15 \mathrm{cc}$ was injected via syringe into each pellet. All the odorants were colorless, so there was no need to hide the pellets from view.

The pellets were presented to the participants in white glass 60 $\mathrm{ml}$ jars with plastic screw-on lids (O. Berk Co., opal jar No. 2T628PA, white plastic cap No. 58400PV). The jars were washed and reused as necessary, but the lids were disposed of after use with one odorant, as were the pellets. Four jars of each odorant were prepared, so that no jar was presented twice consecutively. Only one sniff was allowed per jar for each trial.

\section{Experimental Design}

The task for all participants was to discriminate between pairwise presentations of the seven odors listed above, which constituted the target set. All experimental training took place one day before the discrimination trials, at approximately the same time of day. The four experimental groups differed in their preliminary experience as follows:

Group $C_{N}$. Group $C_{N}$ (control, no treatment) received no special experience with the target items before testing. The participants in this group came in for only a discrimination session.

Group L. Group L (label training) were trained to label the seven target stimuli the day immediately before discrimination testing. On the 1st pass through the stimuli, these participants provided intuitive labels for the target stimuli; they then practiced for 11 additional passes through the stimuli. The participants labeled each stimulus, the only provision being that they felt they could use this label reliably throughout the session. They were then drilled by the experimenter for the remaining passes and provided with corrective feedback. Each stimulus was presented in a random position within each pass only once.

Group P. Group P (profiling task) performed a profiling task for a period equal to the extra time Group $L$ participants spent in training (approximately $1 \mathrm{~h}$ ). The participants in this group profiled the quality of each target odor for about $8 \mathrm{~min}$, by smelling each substance and providing 5-point-scale numerical ratings on a 146-item adjective list designed by Dravnieks (1981). Participants smelled each odor 12 times in the $8 \mathrm{~min}$ allowed per odorant (the number of sniffs that the participants in Group L experienced). The participants were instructed to work in silence and not to use overt verbal labels. When the 8-min period was over for one odorant, the profiling of the next odorant was started when the participant was ready. The odorants were presented in random order to each participant.

Group $C_{L}$. Group $C_{L}$ (control, label learning) participants were trained to label seven different odors, following the procedure used for training Group L participants. The purpose of this group was to assess any possible advantage that the participants might gain by engaging in "generic" odor-name training and/or by simply participating in the experiment. The following odorants were used for this purpose: ethyl butyrate, $0.05 \%$ (Pfaltz \& Bauer); p-anisaldehyde, 0.41\% (Macalaster Bicknell); trans-2-hexenal, $0.02 \%$ (Pfaltz \& Baver); lavandin abrialis, $0.02 \%$ (IFF); methyl propionate, $1.22 \%$ (Eastman); pinene, $0.05 \%$ (Macalaster Bicknell); pyridine, $0.06 \%$ (Alfa). All were matched for intensity and chosen according to the criteria used to select target odors.

\section{Procedure}

A total of 42 stimulus pairings was used in the discrimination task. Both orderings were tested to ensure that order effects, if any, would be controlled for. For example, for discriminating octanal from butyl alcohol, the pairings occurred in the following order: octanal versus butyl alcohol, and butyl alcohol versus octanal. Half of the trials consisted of a target paired with itself, so that 42 same pairings were randomly interspersed with the 42 different pairings, for a total of 84 trials per session.

A two-interval, same-different task was used (2I-AX). For mnemonic purposes, in this experiment the A stimulus will be denoted as target and the $X$ notation will be kept to represent the stimulus presented in the second interval. The participants smelled the target immediately before each $\mathrm{X}$ stimulus; this minimized memory demands.

In a 2I-AX design, $d^{\prime}$ has been shown to underestimate discrimination performance and result in receiver-operating characteristic (ROC) curves with nonunit slopes when plotted in double probability coordinates (Macmillan, Kaplan, \& Creelman, 1977). The 
recommended method of analysis is to construct ROC curves, observe their shapes, and choose an unbiased estimate of receiver performance. Therefore, the participants were instructed to report confidence ratings along with their same or different response. Such ratings can be used to construct $R O C$ curves. In the case of $R O C$ curves that violate the assumptions of normally distributed signal and signal + noise distributions of equal variance, a nonmetric estimate of area under the ROC curve such as $A^{\prime}$ should be used in lieu of $d^{\prime}$ (Gescheider, 1984; Pollack \& Norman, 1964).

The discrimination trials took place as follows: Two jars containing odorant were placed before the participant. The participant opened the first, smelled it, then opened the second, responded "same" or "different," and finally provided a confidence rating between 1 (not confident) and 5 (very confident). Each subsequent trial began after a 20 -sec intertrial interval, a sufficient time to avoid adaptation, as had been demonstrated in preliminary experimentation with these odorants. Approximately 2-3 sec elapsed between sniffs of the target and the $X$ odorant.

\section{Preliminary Training}

\section{Results}

The purpose of teaching the Group $L$ participants to label the target stimuli was to increase target familiarity. Therefore, to argue that familiarity might improve discriminability, it was first necessary to demonstrate that the participants showed improved ability to label the target stimuli after training.

The participants in Group L improved from a mean of between three and four correct labels to approximately five by the end of training. A 2-way ANOVA for group and block number showed a difference between Groups $\mathrm{L}$ and $C_{\mathrm{L}}$ in terms of labeling performance $[F(1,26)=4.16$, $p=.05]$, an effect of block $[F(10,260)=2.85$, $p=.002]$, and no interaction $[F(10,260)=1.68$, $p>.05]$.

The odors used to train the Group $\mathbf{C}_{\mathbf{L}}$ participants were labeled more easily than the target odors. During Block 1, participants in this group correctly labeled approximately one more odor than did participants trained on the target stimuli. This is attributed to the odorant selection process. Target odors were selected to be of low initial familiarity and neutral pleasantness. Of the 30 library items, the optimal 7 were chosen for targets. The control stimuli used for Group $C_{L}$ were not perfectly matched to the 7 target stimuli. This selection of stimuli did, however, ensure that all the participants entered the discrimination experiment on the following day at the same level of proficiency for their respective odorant sets.

The number of correct labels given during Block 1 provided an approximation of the inherent labelability of the target stimuli. Therefore, the participants who received no label training could be expected consistently to label three or four of the stimuli (actual mean = 3.7). Hence, label training endowed the participants with improved ability to label the targets by approximately $30 \%$.

\section{Receiver-Operating Characteristic Curves}

The confidence ratings of discrimination judgments allowed construction of ROC curves, which were based on aggregate confidence ratings pooled over all participants within a group. Adjacent confidence intervals were pooled so that responses falling into, for example, the categories "same, 5" and "same, 4" were pooled to form one confidence interval; "same, 3" and "same, 2" were pooled to form the next; and so on. This procedure resulted in estimates for four criterion levels. The ROC curves for each group appear in Figure 1.

The ROC curves for Groups $L$ and $P$ both appear to deviate somewhat, in similar fashion, from some common assumptions of signal-detection theory. The curves appear to be slightly concave downwards, suggesting signal and signal + noise distributions that may deviate from normal. The reasons for this are unclear. Therefore, a more conservative measure of sensitivity, such as $A^{\prime}$, which makes fewer assumptions about underlying distributions, is more appropriate (Gescheider, 1984; Pollack $\&$ Norman, 1964). $A^{\prime}$ provides an appropriate metric-free measure of sensitivity that can be readily translated into familiar terms-that is, percent correct in a twoalternative, forced-choice task (2-AFC).

All further analyses of the discrimination data will be based on the following formula:

$$
A^{\prime}=1 / 2+\frac{[p(\text { hits })-p(\text { false alarms })][1+p(\text { hits })-p(\text { false alarms })]}{[4 p \text { (hits) })[1-p \text { (false alarms })]}
$$

A hit was scored when a participant correctly responded "same" when presented with identical stimuli. A false alarm was scored when a participant incorrectly responded "same" to two different stimuli.

\section{Discrimination Performance}

Average group $A^{\prime}$ ranged from 0.81 for Group $\mathrm{C}_{N}$, to 0.94 for Group L. This range of scores shows that the

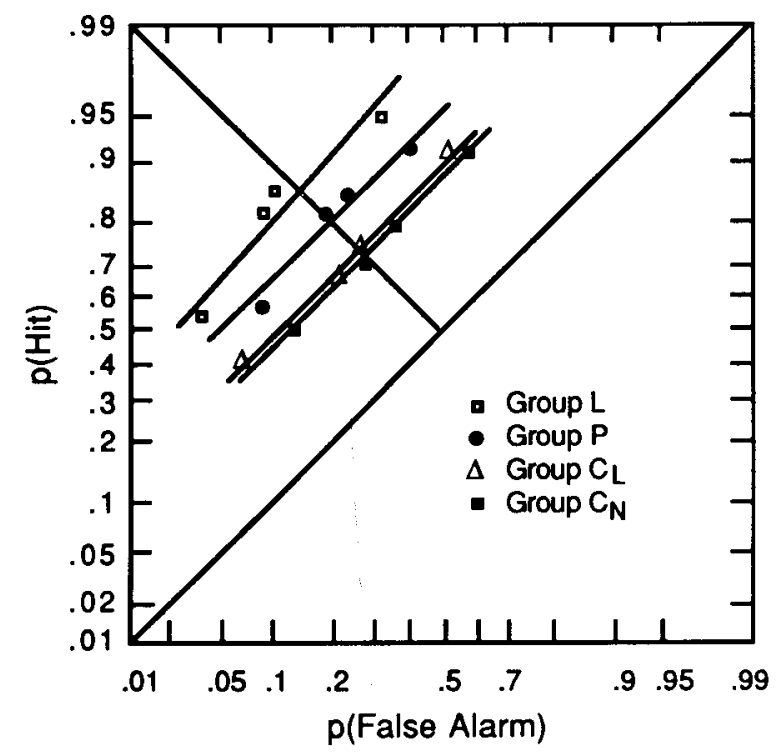

Figure 1. Receiver-operating characteristic curves for the four experimental groups. 


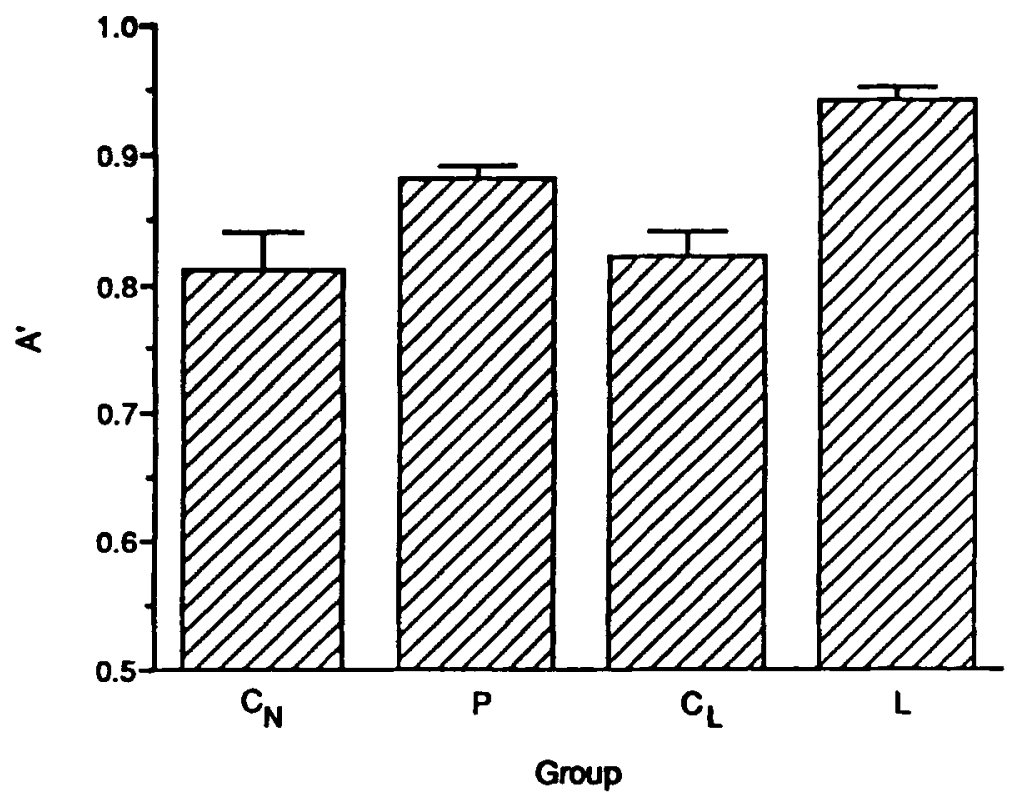

Figure 2. $A^{\prime}$ for each of the four experimental groups $+1 S E M$.

task was somewhat challenging, since the maximum value $A^{\prime}$ can attain is 1.0 , but not too difficult, with $A^{\prime}$ well above chance performance of .50 .

Figure 2 depicts $A^{\prime}$ for each of the four experimental groups. An ANOVA revealed significant between-group differences $[F(3,48)=11.34, p<.0001]$, no effect for sex of the participant $[F(1,48)=0.774$, n.s.), and no group by sex interaction $\left[F(3,48)=1.27\right.$, n.s..$^{1}$ Newman-Keuls pairwise comparisons showed that each group difference depicted in Figure 2 reached significance of at least $p<.05$, except in the case of the comparison between Groups $C_{N}$ and $C_{L}$, the two control groups.

Group $\mathrm{L}$, the group that was trained to label the target odors prior to discrimination testing, outperformed each of the other experimental groups in the discrimination task. Group P, the group whose task was to profile the quality of the targets prior to discrimination testing, did not score as well as Group $L$, but exhibited better ability to discriminate than did either of the other two control groups, $C_{L}$ or $C_{N}$. Group $C_{L}$, trained to label seven nontarget odors, performed no better than Group $\mathrm{C}_{\mathrm{N}}$, which received no prior experimental experience. The influence of experimental manipulation on discrimination performance can therefore be summarized: [label training on targets] $>$ [profile experience on targets] $>$ [label training on controls $=$ no prediscrimination experience].

\section{Labeling Ability and Discrimination}

Although the sex of the participants did not prove to be an important determinant of discrimination performance, another measure of individual differences that may be derived from these data is a participant's overall ability to label an odor. If the hypothesis that label learning improves discriminability is correct, then it might follow that participants best able to label odors would also be better able to discriminate between odors.

The measure for labeling ability was the mean number of correct identifications for the last three label learning blocks for both Group $C_{L}$ and Group $L$ participants. The Pearson product-moment correlation between $A^{\prime}$ and labeling ability for the last three blocks equalled 0.63 $(p<.05)$ for Group $\mathrm{C}_{\mathrm{L}}$, and $0.52(.05<p<.10)$ for Group L. Therefore, regardless of group membership, the participants' abilities to discriminate and label were moderately correlated. Eskenazi, Cain, and Friend (1986) reported a correlation of 0.48 for similar olfactory tasks.

\section{Discussion}

Experiment 1 demonstrated that experience with the specific target odors prior to testing enhanced the ability to discriminate between them. Furthermore, the type of experience determined the amount of improvement in discrimination performance.

The two control groups, $\mathrm{C}_{\mathrm{N}}$ and $\mathrm{C}_{\mathrm{L}}$, performed at equal $A^{\prime}$ scores of approximately 0.82 (equal to $82 \%$ in a 2-AFC task), demonstrating that experience in performing the pretraining tasks did not improve discrimination. This level is presumably the base level of discrimination performance for the seven odorants. Providing the participants in this experiment with the opportunity to smell the odors ahead of time and rate the applicability of the 146 adjectives improved performance to an $A^{\prime}$ of 0.88 . Providing participants in this experiment with label training on the target odors ahead of time improved performance further, to an impressive $A^{\prime}$ of 0.94 . The present results, though, do not allow us to distinguish between the effects of profiling an odor and of simply smelling it before discrimination testing. Perhaps another control 
group, allowed only to smell the odors for the same amount of time as did Groups $\mathrm{L}$ and $\mathrm{P}$, might have also shown improved ability to discriminate relative to Group $\mathrm{C}_{\mathrm{N}}$.

Gibson's (1969) suggestion that labels might assist discrimination by improving memory is consistent with the present results, because the present experiment utilized a two-interval discrimination. Because the odorants were presented in two intervals, the discrimination may have relied somewhat on memory. Indeed, verbal encoding has been shown to improve short-term odor-recognition memory for durations as short as $26 \mathrm{sec}$ (Walk \& Johns, 1984). In the present experiment, 2 to $3 \mathrm{sec}$ separated the stimuli. Therefore, even though $3 \mathrm{sec}$ would represent an extreme test of short-term retention of odors, it is not possible to rule out the possibility that improved verbal encoding led to improved short-term recognition of the target odorant.

Gibson (1969) also suggested that learning to label a stimulus might highlight its discriminative features. A discriminative-features hypothesis would argue that teaching one to label an odor forces one to attend to the attributes of the stimulus that make it unique. In order to identify a stimulus correctly, one must form an accurate perceptual representation of that stimulus and be aware of the properties that make it unique within the entire stimulus array. The participants who were trained to label the target stimuli were forced to attend to the stimulus attributes that made each odor unique. The participants who profiled the stimuli were never faced with the task of having to provide a unique perceptual identity for each target stimulus.

Although a two-interval discrimination task offers no resolution between a memory-trace account of improved discrimination and a discriminative-features account, two odors can be presented simultaneously in mixture. Simultaneous presentations of odors would allow one to examine whether or not the results reported in Experiment 1 extended to the perceptual processing of odor mixtures.

\section{EXPERIMENT 2}

Experiment 2 was designed to investigate perceptual learning in olfaction from a perspective somewhat different from, although convergent with, that of Experiment 1. Experiment 2 was an attempt to relate the odor knowledge that a participant brought into the experiment to performance on a discrimination task that involved the simultaneous presentation of odors. In this task, however, each target odor was paired either with itself or with a transform of itself. Each transform consisted of target odor plus a less intense familiar or unfamiliar contaminant. Since odor familiarity appears to vary greatly from person to person, in Experiment 2 stimulus sets were individually selected for each participant.

This experiment was intended to investigate whether familiarity with an odor might enhance the ability to detect its contamination, and whether familiarity with the contaminant might assist in its discrimination against the background of a stronger odor. Each transform, however, was a mixture (target + contaminant), so that in order to perform the discrimination successfully, the participant had to determine accurately the nature of the transform, thereby discriminating between odors presented in parallel.

\section{Method}

\section{Participants}

Five males and five females participated in Experiment 2. All were between the ages of 20 and 30 (mean age $=24$ ) and were paid for their time.

\section{Stimulus Selection}

The participants first smelled each of the $\mathbf{3 0}$ matched-intensity odors in the odor library during a preliminary evaluation session. The entire set was presented twice. During the first pass through the set, the participants rated pleasantness on a scale ranging from -5 to +5 , rated familiarity on a scale from 0 to 10 , and finally provided a label for the odor. The participants were instructed to provide the most appropriate label possible and told that they would be asked to label the odors a second time on a second pass through the set. During the second pass, the participants labeled the odors again, trying to use the labels they gave during the first pass. Twenty seconds lapsed between odorant presentations, and approximately 2 min separated the two passes through the library.

Each participant's ratings were later evaluated, in order to choose the stimuli for discrimination testing. The stimuli were defined as either Familiar $(F+)$ or Unfamiliar $(F-)$. The $F+$ stimuli were those given high familiarity ratings and labeled consistently during both runs through the odor library (consistent labeling of odors provides a practical estimate of encodability; see Rabin \& Cain, 1984). The F- stimuli were chosen on the basis of low familiarity ratings and inconsistent labeling.

The target set contained three $\mathrm{F}+$ stimuli and three $\mathrm{F}-$ stimuli. The six targets were approximately equal in moderate perceived intensity. Each transform was composed of a target + another less intense target (either $\mathrm{F}+$ or $\mathrm{F}-$ ) as a contaminant; the contaminants were chosen so that they equaled 2 on the intensity scale.

In practice, it was not always possible to select odorants to represent extremes of familiarity, since pleasantness and familiarity tend to be correlated. In this experiment too, familiarity and pleasantness showed a positive correlation (average $r=0.43$, $p<.05$ ). Compromises were necessary, to ensure that the degrees of pleasantness of the $\mathrm{F}+$ and $\mathrm{F}-$ stimuli were about equal. The odorants chosen to be $\mathrm{F}+$ and $\mathrm{F}-$ stimuli differed in rated familiarity $(t=7.42, p<.0001)$ and did not differ in their rated pleasantness $(t=1.85, p>.05)$.

Table 1 shows the percentage of cases in which each stimulus was determined to be either $F+$ or $F-$. Bezaldehyde, for instance, was chosen to be an F+ stimulus $10 \%$ of the time, and never used as an F- stimulus. Octanal, on the other hand, served as an F+ stimulus $7 \%$ of the time, and served as an F- stimulus $13 \%$ of the time. Hence, it was familiar to some participants, and unfamiliar to others. Such a pattern, which occurred not infrequently, highlights the individuality of odor experience.

\section{Stimulus Delivery}

Stimulus delivery was accomplished as in Experiment 1, with a similar jar-and-pellet system. In Experiment 2, two pellets were used in each jar. One was impregnated with the target odorant, the second-when appropriate-with the contaminant. A dummy pellet containing mineral oil diluent was included with the target pellet for target-only presentations. This delivery method prevented the 
mixing of the two chemicals in the liquid phase and assured that mixing would occur in the vapor phase in the headspace of a jar.

\section{Procedure}

Table 2 shows how the target transforms were prepared for each participant. Each row of entries was used only as the $X$ pool for the associated target odorant, so that, for example, Target 3 was only tested against its transforms, $3+1$ through $3+6$. Note that the stimuli were not the same for each participant, because they were chosen to represent a range of familiarity for each participant. In the actual testing, trials were run in both possible orders [e.g., 3 vs. $(3+1)$, and $(3+1)$ vs. 3 ]. In addition, an equal number of same trials were randomly interspersed among different trials. Same trials consisted of two identical target items.

Each discrimination trial took place exactly as in Experiment 1. The participants in Experiment 2 were told that they would be presented either with stimuli that were identical, or with two stimuli that were different because one had an extra odor added. Each session included all the F + and F-transforms, consisted of 128 trials, and lasted approximately $1.5 \mathrm{~h}$. The discrimination trials took place within 1 week of the evaluation session.

\section{Analysis of Responses}

A hit was scored if a participant correctly called identical targets "same." A false alarm was scored if a pairing of a target and its transform was called "same." The false alarm rate represents the rate at which participants were unable to detect the contaminant against the background of the target odor. $A^{\prime}$ was used as the performance measure, so that the results could be compared to those of Experiment 1. Four separate A's were calculated for each par-

Table 1 Odorant Distribution into Categories

\begin{tabular}{lcc}
\hline & \multicolumn{2}{c}{ Percent of Each } \\
\multicolumn{1}{c}{ Odorant } & Fategory Accounted For \\
\cline { 2 - 3 } Amyl Acetate & 7 & F- \\
Amyl Butyrate & 3 & 0 \\
p-Anisaldehyde & 3 & 3 \\
Anisole & 0 & 0 \\
Benzaldehyde & 10 & 7 \\
Butyl Alcohol & 0 & 0 \\
n-Butyric Acid & 0 & 7 \\
l-Carvone & 13 & 0 \\
Cineole & 3 & 0 \\
Cinnamyl n-Butyrate & 3 & 3 \\
Ethyl Butyrate & 7 & 3 \\
Ethyl Propionate & 0 & 0 \\
Ethyl Valerate & 3 & 7 \\
tr-2-Hexenal & 3 & 3 \\
cis-3-Hexen-1-ol & 7 & 10 \\
n-Hexyl Salicylate & 0 & 7 \\
Hydroxycitronellal & 0 & 3 \\
Isovaleric Acid & 0 & 10 \\
Lavandin Abrialis & 7 & 0 \\
Linalool & 3 & 0 \\
Linanlyl Acetate & 3 & 10 \\
Methyl Disulfide & 0 & 10 \\
Methyl Propionate & 0 & 0 \\
Octanal & 7 & 0 \\
b-Phenethyl Alcohol & 3 & 13 \\
sec Phenethyl Alcohol & 0 & 0 \\
Pinene & 7 & 0 \\
Propyl Butyrate & 7 & 3 \\
Pyridine & 0 & 0 \\
trans-2-Decen-1-al & 0 & 0 \\
\hline F+ & & 0 \\
\hline
\end{tabular}

Note $-F+=$ familiar; $F-=$ unfamiliar.
Table 2

Transformation Table

\begin{tabular}{ccccccc}
\hline Target & $1(\mathrm{~F}+)$ & $2(\mathrm{~F}+)$ & $3(\mathrm{~F}+)$ & $4(\mathrm{~F}-)$ & $5(\mathrm{~F}-)$ & $6(\mathrm{~F}-)$ \\
\hline $1(\mathrm{~F}+):$ & - & $1+2$ & $1+3$ & $1+4$ & $1+5$ & $1+6$ \\
$2(\mathrm{~F}+):$ & $2+1$ & - & $2+3$ & $2+4$ & $2+5$ & $2+6$ \\
$3(\mathrm{~F}+):$ & $3+1$ & $3+2$ & - & $3+4$ & $3+5$ & $3+6$ \\
$4(\mathrm{~F}-):$ & $4+1$ & $4+2$ & $4+3$ & - & $4+5$ & $4+6$ \\
$5(\mathrm{~F}-):$ & $5+1$ & $5+2$ & $5+3$ & $5+4$ & - & $5+6$ \\
$6(\mathrm{~F}-):$ & $6+1$ & $6+2$ & $6+3$ & $6+4$ & $6+5$ & - \\
\hline
\end{tabular}

Note $-F+=$ familiar; $F-=$ unfamiliar.

ticipant, one for each possible combination of target and contaminant.

Two separate hit rates were used to calculate the appropriate $A^{\prime}$. One hit rate was calculated for each of the two target types, F+ and F - by pooling the total hits for the three F + odors together, and for the three F- odors together, and dividing by the respective number of same trials.

Together with these two hit rates, four false alarm rates were calculated, corresponding to each of the four possible combinations of target and contaminant. For example, for an F+ target and an $\mathrm{F}$ - contaminant (abbreviated as $\mathrm{F}+\mathrm{F}-$ ), the false alarm rate was based on all trials in which an F+ target was mixed with an Fcontaminant.

\section{Results}

\section{Target and Contaminant as Determinants of Discriminability}

Figure 3 presents a summary of discrimination performance with respect to the four possible pairings of target and contaminant $(F+F+, F+F-, F-F+, F-F-)$. Both target type and contaminant type appeared to influence the difficulty of the discrimination.

An ANOVA was performed with target type (F+ or $\mathrm{F}-$ ) and contaminant type $(\mathrm{F}+$ or $\mathrm{F}-)$ as repeated measures across the 10 participants. Both target type $[F(1,9)$ $=16.03, p=.003]$ and contaminant type $[F(1,9)=$ $10.91, p=.009]$ influenced discrimination performance with no significant interaction between them $[F(1,9)=$ 0.59 , n.s.].

The results, then, appear to suggest that a familiar target is more likely to be discriminated from a contaminated version of itself than an unfamiliar target is. Furthermore, a familiar contaminant is more likely to be apparent to an observer than an unfamiliar one.

\section{Labeling Ability and Discrimination}

As in the case of Experiment 1, it was possible to extract a measure of overall odor-labeling ability for each participant and relate it to overall discrimination performance. For Experiment 2, this measure consisted of the number of consistently labeled odors in the preliminary evaluation session. This number ranged from a low of 5 to a high of 14 , out of the 30 -item odor library. Overall $A^{\prime}$ in Experiment 2 ranged from a low of 0.70 to a high of 0.78 (mean $=0.75, S E M=0.008$ ). The participant who scored the low of 0.70 labeled only 5 of the odors consistently in the evaluation session, while the two participants who scored 0.78 labeled 10 and 14 odors consistently. The Pearson product-moment correlation be- 


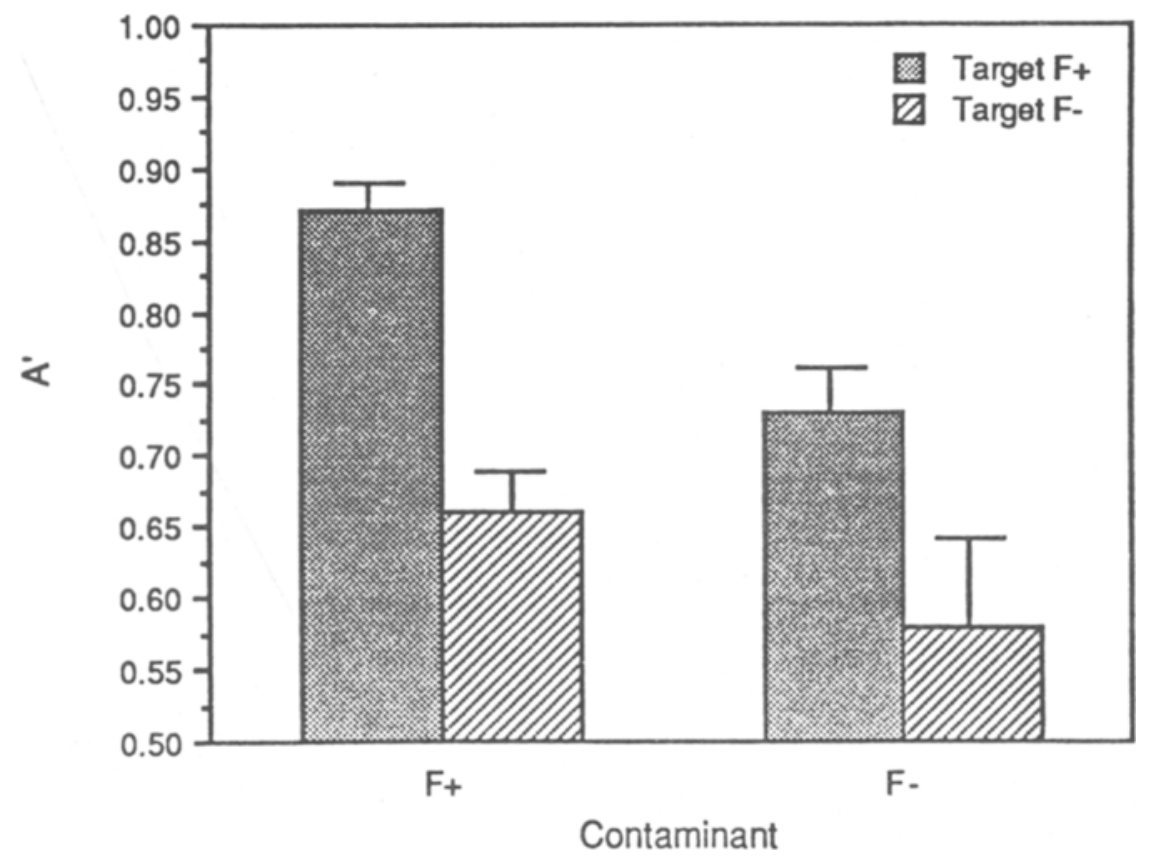

Figure 3. Discrimination performance for the four combinations of target and contaminant stimuli.

tween $A^{\prime}$ and number of consistently labeled odors equalled $0.53(p=.12)$. Although this correlation equals almost exactly that obtained in Experiment 1 between $A^{\prime}$ and number correctly labeled for the last three blocks, it is not significant, due to the fewer degrees of freedom in the present experiment. Nevertheless, the direction and the approximate magnitude of the relationship are consistent with those in Experiment 1 and in other published reports.

\section{Discussion}

Two questions were phrased in the introduction to Experiment 2: First, does familiarity with an odor enhance the ability to detect whether it is contaminated with anything else? And second, does familiarity with the contaminant assist the discrimination of it against the background of a stronger odor?

\section{Target Familiarity}

High target familiarity, as indexed by numerical rating of familiarity and the ability to label it consistently, facilitated the detection of a contaminant's presence in the odor. One explanation of this result is that the participants possessed a more finely tuned perceptual representation of a familiar stimulus and were better able to judge whether or not another stimulus, a transform of the familiar one, violated the perceptual boundaries of the first. According to this interpretation, accurate labeling indicated the presence of a unique perceptual category congruous with discriminative capacity. This finding was in accord with speech research suggesting that perceptual categories are subject to the effects of training and experience (Carney,
Widen, \& Viemeister, 1977; Pisoni, Aslin, Perey, \& Hennessy, 1982).

\section{Contaminant Familiarity}

The familiarity of the contaminant also influenced the ease of this discrimination task. A simple memorytrace-comparison explanation of this result seems less tenable, due to the absence of contaminant in one of the two sampling intervals constituting a trial. In this case the contaminants, whether familiar or unfamiliar, were present in only one sampling interval. The detection of their presence, then, is most parsimoniously interpreted as a matter of stimulus salience, rather than memory-trace comparison. This result implies that the participants could not detect the contaminant as readily if it was unfamiliar.

In fact, the false alarm rate for the F- contaminants was above $45 \%$, suggesting a serious detection problem. The false alarm rate for $\mathrm{F}+$ contaminants averaged about $30 \%$. It seems, then, that the familiar contaminants were good stimuli in the sense that Garner (1974) uses the term, implying that they exist in unique classes and share few overlapping features with other stimuli. Bad stimuli, such as unfamiliar ones, possess few unique perceptual features and therefore blend in more readily against a stronger background.

\section{Implications for Mixture Perception}

The results of Experiment 2 demonstrate that the ability to detect the individual components of an odor mixture is not a simple function of stimulus intensities. If stimulus intensity were the only determinant of the ability to resolve an odor mixture, then the different types 
of contaminant would have influenced discrimination equally.

Laing and Willcox (1983) performed a major investigation of both odor quality and intensity for several binary odor mixtures. The participants in their study profiled the odor quality of mixtures and individual components, using an adjective-list profiling technique. Laing and Willcox reported that (1) mixtures composed of two odors of equal perceived (unmixed) intensity were profiled as a combination of the separate odor profiles, even though some features were missing in the mixture; and (2) very small changes in the ratio of the perceived intensities of the individual components shifted the perception of the mixture largely in the direction of the stronger component.

Laing and Willcox (1983) noted in their study that their profiling technique was not necessarily sensitive enough to detect whether participants could actually discriminate between the binary mixtures even though the profiles may have been similar. Their results implied that when the perceived intensity of one of the mixture components increased over that of the other, the profiling of the stimulus shifted categorically in the direction of the more intense. The results of the present experiment suggest that discrimination may indeed provide a picture of perception different from that provided by profiling alone, and they supplement the results of Laing and Willcox by suggesting that cognitive attributes of odor stimuli will influence the detection of mixture components. The results of Experiment 2 are likely to be general across odors, since each participant discriminated among a different stimulus array chosen according to psychological, not chemical, variables.

\section{GENERAL DISCUSSION}

The two experiments reported here approached the problem of perceptual learning in olfaction from two different but convergent perspectives. Experiment 1 showed that experimentally increasing the familiarity of simple target odors resulted in an improved ability to discriminate between them. Experiment 1, however, could not resolve which of two possible sources was responsible for this improvement: memory trace or distinctive features. Experiment 2 showed that odor experience garnered over a lifetime could be put to use in performing a relatively more difficult discrimination/mixture-resolution task. These two experiments made different demands on the participants and were not equally difficult. The mean $A^{\prime}$ for Experiment 1 equalled $0.83(S E M=0.01)$, and for Experiment 2, $0.75(S E M=0.01)$; their results, however, are in close accord.

Another piece of converging evidence supports the idea that relative olfactory discrimination is not independent of familiarity: the result that measures of individual differences in labeling ability correlate with performance on the discrimination tasks. The better a person seems to be at labeling odors, the better he or she may be at discriminating between them. This finding, however, is relatively circumstantial, since the measure of labeling ability might only indicate general olfactory aptitude, verbal ability, or even, perhaps, intelligence. Nonetheless, the correlation is consistent with the more general conclusion gained from these experiments: cognitive variables may influence our ability to perform olfactory discriminations.

The mechanism by which familiarity might assist discrimination is probably a combination of two processes, as suggested by Gibson (1969). Experiment 1 involved a memory component, since the comparison of the to-bediscriminated stimuli was successive. Rabin and Cain (1984) demonstrated that long-term memory for odors depends in part on familiarity. Walk and Johns (1984) extended this conclusion to short-term (26-sec) retention of olfactory information. Therefore, to the extent that the discrimination task relied on a memory-based representation of the stimulus, a memory-trace account might explain the data.

Increased stimulus familiarity might also assist discrimination by forcing the participant to attend to the discriminative features of a stimulus (Walk, 1978). In Experiment 2, the participants performed as if a familiar odor were a better stimulus, more salient and more detectable, than an unfamiliar one. The results of Experiment 2 lend themselves to a distinctive-features interpretation in which more familiar stimuli possess sharper categorical boundaries.

Although discrimination performance in both of these experiments appeared to benefit from increased familiarity with the stimuli, discrimination between unfamiliar stimuli was still above chance performance. Clearly, familiarity alone is not necessary to distinguish between odors. For long-term odor recognition, too, human beings seem able to use what might be termed osmorphic (or odor-iconic) information-processing strategies (Rabin \& Cain, 1984). Pleasantness, too, appears to play a role in olfactory discrimination (Rabin \& Cain, in press).

The present finding helps to explain why perfumers and odor experts are able to treat odor mixtures analytically, whereas the layperson may be unable to determine the components of an odor mixture. Endowing a layperson with a perfumer's experience would make subtle mixture components more salient stimuli. Although these experiments tested only a relatively small number of different odor qualities, the brief training period in Experiment 1 suggests that over a longer period, these results might be extended to cover a much larger odor library.

It is interesting that the results of Experiment 1 extend to Experiment 2 as well. This implies that the cognitive processing of odor mixtures operates according to principles not unlike those that apply to single stimuli. It also implies that simple psychophysical measures, based on group averages, may not provide the most veridical mea- 
sure of odor-mixture quality, since the quality perceived will probably vary from individual to individual, depending on that person's past olfactory history.

\section{REFERENCES}

CAIN, W. S. (1979). To know with the nose: Keys to odor identification. Science, 203, 467-470.

Carney, A. E., Widen, G. P., \& Viemeister, N. F. (1977). Noncategorical perception of stop consonants differing in VOT. Journal of the Acoustical Society of America, 62, 961-970.

Desor, J. A., BEAUChAMP, G. K. (1974). The human capacity to transmit olfactory information. Perception \& Psychophysics, 16, 551-556.

DravNIEKS, A. (1981). Odor profiling exercise. American Society for Testing and Materials, E18.04.12.

Engen, T., \& Praffmann, C. (1960). Absolute judgments of odor quality. Journal of Experimental Psychology, 59, 214-219.

ENGEN, T., Ross, B. M. (1973). Long-term memory of odors with and without verbal descriptors. Journal of Experimental Psychology, 100, 221-227.

EsKenazi, B., CAIN, W. S., FRIEND, K. (1986). Exploration of olfactory aptitude. Bulletin of the Psychonomic Society, 24, 203-206.

GARNER, W. R. (1962). Uncertainty and structure as psychological concepts. New York: Wiley.

GARNER, W. R. (1974). The processing of information and structure. Potomac, MD: Erlbaum.

Gescheider, G. A. (1984). Psychophysics: Method, theory and application (2nd ed.). Hillsdale, NJ: Erlbaum.

Gibson, E. J. (1969). Principles of perceptual learning and development. Englewood Cliffs, NJ: Prentice Hall.

Gibson, E. J., WALK, R. D. (1956). The effects of prolonged exposure to visually presented patterns on learning to discriminate them. Journal of Comparative and Physiological Psychology, 49, 239-242.

LAING, D. G., Willcox, M. E. (1983). Perception of components in binary odour mixtures. Chemical Senses, 7, 249-264.

LAWLESS, H. T., \& CAIN, W. S. (1975). Recognition memory for odors. Chemical Senses \& Flavor, 1, 331-337.

Macmillan, N. A., Kaplan, H. L., Creelman, C. D. (1977). The psychophysics of categorical perception. Psychological Review, 84, 452-471.

MURRAY, F. S., \& LEE, T. S. (1977). The effects of attention-directing training on recognition memory task performance of three-year-old children. Journal of Experimental Child Psychology, 23, 430-441.

Pick, A. D. (1965). Improvement of visual and tactual form discrimination. Journal of Experimental Psychology, 69, 331-339.

Pisoni, D. B., Aslin, R. N., Perey, A. J., \& Hennessy, B. L. (1982). Some effects of laboratory training on identification and discrimination of voicing contrasts in stop consonants. Journal of Experimental Psychology: Human Perception \& Performance, 8, 297-314.

PollaCK, I. (1952). The information of elementary auditory displays. Journal of the Acoustical Society of America, 24, 745-749.

Pollack, I., \&ORMan, D. A. (1964). A non-parametric analysis of recognition experiments. Psychonomic Science, 1, 125-126.

RabiN, M. D., \& CAIN, W. S. (1984). Odor recognition: Familiarity, identifiability, and encoding consistency. Joumal of Experimental Psychology: Learning, Memory, \& Cognition, 10, 316-325.

RabiN, M. D., \& CAIN, W. S. (in press). Attention and learning in the perception of odor mixtures. In D. G. Laing, W. S. Cain, R. L. McBride, \& B. W. Ache (Eds.), Perception of complex tastes and smells. Sydney, Australia: Academic Press.

Robinson, J. S. (1955). The effect of learning verbal labels for stimuli on their later discrimination. Joumal of Experimental Psychology, 49, 112-114.

Schiffman, S. S., Robinson, D. E., \& Erickson, R. P. (1977). Multidimensional scaling of odorants: Examination of psychological and physiochemical dimensions. Chemical Senses \& Flavor, 2 , 375-390.

WalK, R. D. (1978). Perceptual learning. In E. C. Carterette \& M. P. Friedman (Eds.), Handbook of perception: Vol. 9. Perceptual processing (pp. 257-297). New York: Academic Press.

WALK, H. A., \& JoHNS, E. E. (1984). Interference and facilitation in short-term memory for odors. Perception \& Psychophysics, 36, 508-514.

\section{NOTE}

1. These results are paralleled if the ANOVA is performed using $d^{\prime}$ taken from the tables provided by Macmillan et al. (1977) for 2I-AX tasks. For group: $F(3,48)=17.10, p<.001$; for $\operatorname{sex}: F(1,48)=2.00$, n.s.; and for sex $\times$ group interaction: $F(3,48)=0.78$, n.s.

(Manuscript received December 21, 1987; revision accepted for publication June 10, 1988.) 